\title{
miR-21 promotes NLRP3 inflammasome activation to mediate pyroptosis and endotoxic shock
}

\author{
Zhenyi Xue', Qing Xi', Hongkun Liu', Xiangdong Guo ${ }^{1}$, Jieyou Zhang ${ }^{1}$, Zimu Zhang ${ }^{1}$, Yan Li ${ }^{1}$, Guangze Yang ${ }^{1}$, \\ Dongmei Zhou', Huiyun Yang ${ }^{1}$, Lijuan Zhang', Qi Zhang' ${ }^{2}$, Chao Gu' ${ }^{3}$, Juhong Yang ${ }^{4}$, Yurong Da', Zhi Yao', \\ Shuguang Duo ${ }^{5}$ and Rongxin Zhang ${ }^{1,6}$
}

\begin{abstract}
miR-21 is aberrantly expressed, and plays a role in various types of tumors and many other diseases. However, the mechanism of miR-21 in LPS-induced septic shock is still unclear. In this study, we investigated the mechanism of miR21 in LPS-induced pyroptosis and septic shock. Here, we show that miR-21 deficiency inhibited NLRP3, ASC, and caspase-1 expression, as well as inflammasome activation in myeloid cells from both mice and humans. We found that the NF-KB pathway was regulated by miR-21, and that A20 was a direct target of miR-21. Furthermore, miR-21 deficiency inhibited the ASC pyroptosome, which restrained caspase-1 activation and GSDMD cleavage, thereby preventing LPS-induced pyroptosis and septic shock. miR-21 deficiency resulted in an increase in A20, which led to decreased IL-1 $1 \beta$ production and caspase-1 activation. Caspase-1-mediated GSDMD cleavage was consequently decreased, which prevented pyroptosis in LPS-induced sepsis in mice. Our results demonstrate that miR-21 is a critical positive regulator of the NF-KB pathway and NLRP3 inflammasomes in pyroptosis and septic shock via A20. In addition, by analyzing published miRNA expression profiles in the Gene Expression Omnibus database, we found that the miR21 levels in peripheral blood from patients with septic shock were elevated. Thus, miR-21 may serve as a potential treatment target in patients with septic shock.
\end{abstract}

\section{Introduction}

The nucleotide-binding domain, leucine-rich repeatcontaining receptor (NLR) family protein NLRP3 plays key roles in host defense, which can be activated by many pathogen-derived, environmental, and host-derived factors, including bacteria ${ }^{1}$, viruses ${ }^{2}$, fungi ${ }^{3}$, components of dying cells ${ }^{1}$, and crystal particles ${ }^{4-7}$. Secretion of

Correspondence: Yurong Da (dayr@tmu.edu.cn) or Shuguang Duo (duoshuguang@ioz.ac.cn) or Rongxin Zhang (rxzhang@tmu.edu.cn) ${ }^{1}$ Laboratory of Immunology and Inflammation, Department of Immunology, Key Laboratory of Immune Microenvironment and Diseases of Educational Ministry of China, Tianjin Key Laboratory of Cellular and Molecular Immunology, Tianjin Medical University, 300070 Tianjin, China

${ }^{2}$ Institute of Integrative Medicines for Acute Abdominal Diseases, Nankai Hospital, Tianjin, China

Full list of author information is available at the end of the article. These authors contributed equally: Zhenyi Xue, Qing Xi Edited by $\mathrm{G}$. Raschellà proinflammatory IL-1 $\beta$ and IL-18 requires activation of inflammasomes to splice IL-1 $\beta$ and IL-18 precursors into mature forms ${ }^{8}$. Macrophages are the major source of proIL-1 $\beta$ and pro-IL-18, which are generally dependent on caspase-1 for maturation and secretion of the bioactive cytokine. Caspase- 1 activation is controlled by different inflammasomes ${ }^{9}$. In addition, activated caspase- 1 triggers a form of programmed necrosis known as pyroptosis.

Accumulating evidence has suggested that inflammasomes are involved in the pathogenesis of sepsis ${ }^{10,11}$. Inflammasomes trigger pyroptosis in a caspase-1dependent manner ${ }^{12,13}$. In sepsis, pyroptosis promotes pore formation in the plasma membrane, leading to cell swelling and membrane rupture and resulting in leakage of abundant inflammatory factors out of the cell ${ }^{10-15}$. Caspase- 1 and caspases-4/5/11 specifically recognize and

\section{(c) The Author(s) 2019}

(c) (i) Open Access This article is licensed under a Creative Commons Attribution 4.0 International License, which permits use, sharing, adaptation, distribution and reproduction cc) in any medium or format, as long as you give appropriate credit to the original author(s) and the source, provide a link to the Creative Commons license, and indicate if changes were made. The images or other third party material in this article are included in the article's Creative Commons license, unless indicated otherwise in a credit line to the material. If material is not included in the article's Creative Commons license and your intended use is not permitted by statutory regulation or exceeds the permitted use, you will need to obtain permission directly from the copyright holder. To view a copy of this license, visit http://creativecommons.org/licenses/by/4.0/. 
then cleave gasdermin D (GSDMD), which is the core event in pyroptosis ${ }^{16-18}$. Caspase-1 also initiates pyroptosis by cleaving GSDMD. The role of GSDMD in pyroptosis is to trigger lethal sepsis and septic shock ${ }^{18,19}$.

Sepsis is defined as life-threatening organ dysfunction, and is caused by a several dysregulated responses to infection. Septic shock is a subset of sepsis with significantly increased mortality ${ }^{20}$. Many microRNAs (miRNAs) have been identified as key regulators of sepsis and septic shock. A previous study has reported that miR-21 is obviously elevated in the plasma of septic patients and the heart tissue of LPS-induced sepsis mice ${ }^{21}$. Although deregulations of miRNA expression are well described, the pathophysiological role of miRNAs in septic shock has not yet been fully defined.

Due to the key role of the NLRP3 inflammasome in LPS-induced septic shock, we investigated the function of miR-21 in regulating activation of the NLRP3 inflammasome. We found that miR-21 positively regulates activation of NLRP3 inflammasomes by negatively regulating A20 in macrophages, which triggers pyroptosis to promote LPS-induced septic shock through cleavage of GSDMD.

\section{Results}

miR-21 knockout inhibits NLRP3 inflammasome-mediated caspase- 1 activation and IL-1 $\beta$ secretion in macrophages

To investigate the role of miR-21 in activation of the NLRP3 inflammasome, lipopolysaccharide (LPS)-primed BMDMs were challenged with ATP or nigericin. We found that miR-21 knockout (KO) inhibited caspase-1 activation and IL-1 $\beta$ secretion (Fig. 1a, b). Moreover, caspase-1 autoprocessing (Fig. 1c, d) and IL-1 $\beta$ secretion (Fig. 1e, f) caused by NLRP3 inflammasomes significantly decreased during ATP or nigericin treatment for $10 \mathrm{~min}$ and continued to decrease in a time-dependent manner. Induction of LDH-release-based cell death by caspase-1dependent pyroptosis was weakened in miR-21 $1^{-1-}$ macrophages (Fig. 1g, h). The increased reactivity of macrophages to inflammasomes was limited to NLRP3 inflammasomes because stimulation of AIM2 inflammasomes with dsDNA did not induce caspase-1 cleavage, IL-1 $\beta$ secretion, or pyroptosis in wild-type and miR-21 ${ }^{-l-}$ macrophages (Fig. 1i-k). These data clearly indicate that miR-2 1 promotes IL-1 $\beta$ secretion and caspase- 1 activation mediated by the NLRP3 inflammasome in mouse macrophages.

\section{miR-21 regulates NF-KB and NLRP3 inflammasome expression by targeting $A 20$}

To determine the target of miR-21, we further detected the upstream regulatory factors of the NLRP3 inflammasome. In miR-21 ${ }^{-1-}$ macrophages, $A 20$ mRNA expression was increased, and Nlrp3, Asc, Casp1, and Il-1b mRNA expression was decreased (Fig. 2a-e). Western blotting results also showed that the A20 protein level was upregulated, but that NLRP3, ASC, pro-caspase-1, and pro-IL-1b protein levels were downregulated in miR-21 $1^{-1-}$ macrophages (Fig. 2f). To confirm that A20 is a direct target of mir-21, 293 cells were cotransfected with control or miR21 mimic and a dual-luciferase reporter plasmid containing the WT A20 3'-UTR. The results showed that the activity of luciferase in miR-21 mimic-transfected cells was significantly decreased. However, miR-21 mimic had no significant effect on the activity of luciferase in cells transfected with the dual-luciferase reporter plasmid containing an A20 3'-UTR mutated at the mir-21-binding site (Fig. 2g, h).

NLRP3 inflammasome expression levels are regulated by the proinflammatory transcription factor NF- $\kappa B^{22}$. A20 negatively regulates LPS-induced NF- $\mathrm{KB}$ activation, which also influences the secretion of the NF- $\mathrm{KB}$-dependent cytokines IL-6 and TNF in macrophages ${ }^{23-26}$. Inducible activation of NF- $\mathrm{kB}$ depends upon proteasomal degradation of NF- $k B$ protein inhibitors (IkBs) ${ }^{27}$. In this study, we found that there was a dramatic decrease in $\mathrm{p}-\mathrm{IkB} \alpha$ levels following LPS stimulation for different time intervals in miR-21 KO cells, resulting in increased IkB $\alpha$ levels (Fig. 2i). In addition, miR-21 deficiency inhibited the secretion of the NF-kB-dependent cytokines IL-6 and TNF in macrophages (Fig. 2j, k).

We further confirmed the target of miR-21 using lossof-function experiments. The mRNA results showed that A20 knockdown promoted Nlrp3, Asc and Casp1 mRNA expression in miR-21 KO macrophages with or without LPS stimulation (Fig. 3a-d). Similarly, the NLRP3, ASC, and pro-caspase-1 protein levels were enhanced in A20 knockdown macrophages with or without LPS stimulation (Fig. 3e). In addition, caspase- 1 processing and IL-1 $\beta$ secretion were increased in A20 knockdown macrophages with LPS priming and ATP or nigericin stimulation (Fig. 3f, g). Collectively, these data demonstrate that NF- $\mathrm{KB}$ and NLRP3 inflammasome expression was regulated by miR21 through targeting of A20.

\section{miR-21 regulates NLRP3-mediated ASC pyroptosome formation by targeting A20}

In addition to caspase- 1 activation and IL- $1 \beta$ release, the ASC pyroptosome is another hallmark of inflammasome activation, which is considered to mediate caspase-1 activation $^{28}$. In accordance with previous reports, the results of endogenous ASCs immunostaining showed that more than $70 \%$ of macrophages contained the ASC pyroptosome in the WT (70.4\%) and A20 knockdown (80.1\%) group after stimulation with LPS and ATP. Conversely, formation of the ASC pyroptosome was prevented in the miR-21 KO group (14.3\% and 24.2\%) (Fig. 4a-c). In addition, we isolated the ASC pyroptosome using a 


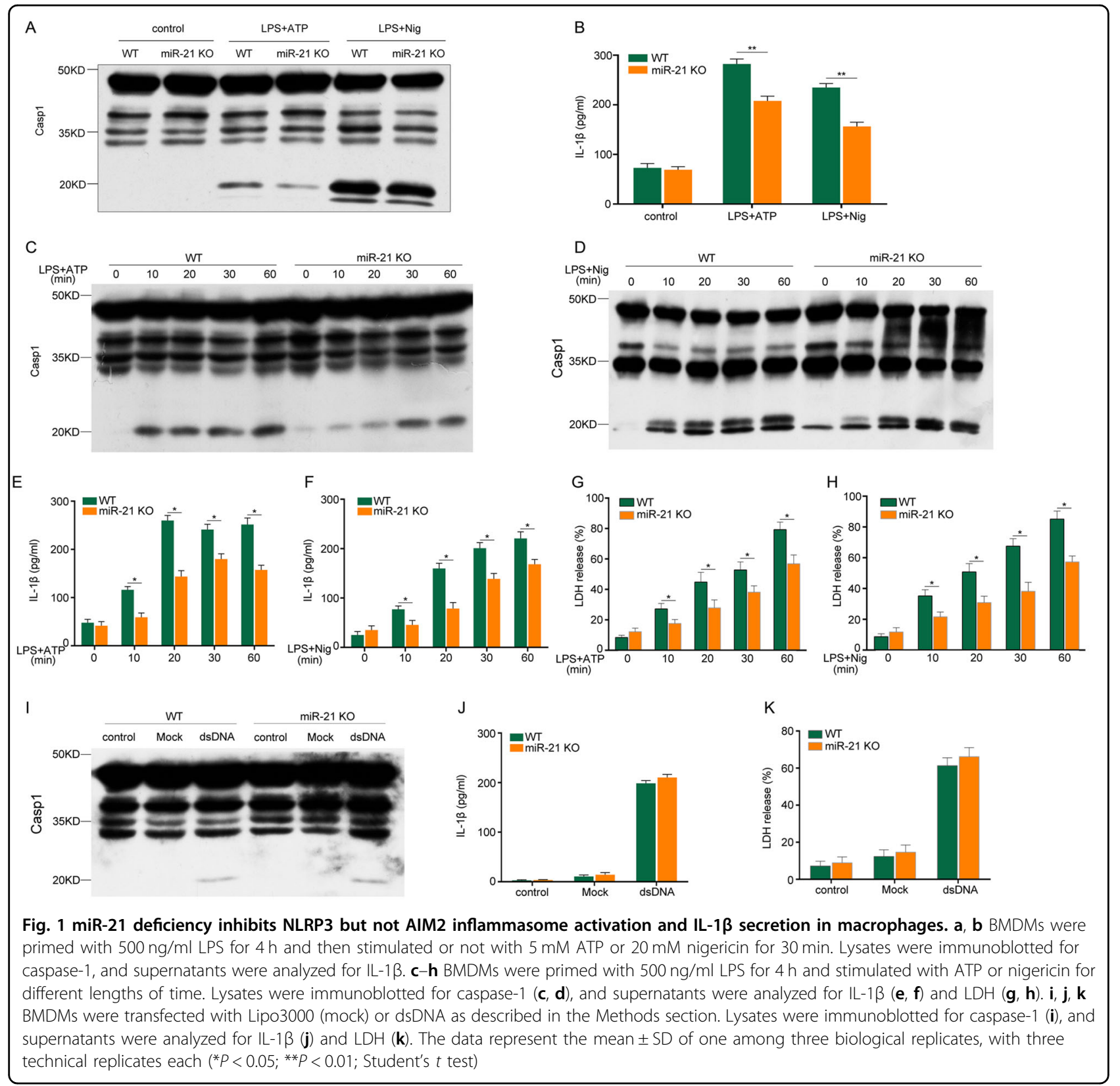

chemical method reported in previous studies ${ }^{29}$. We detected endogenous ASC pyroptosome in BMDMs via western blot analysis. The results showed that ASC proteins were cross-linked by DSS to form ASC dimers, trimers, and oligomers, and were mainly redistributed from the lysates into the pellets in the WT group compared with the miR-21 ${ }^{-1-}$ group (Fig. 4d). Similarly, the capacity to form ASC pyroptosomes increased after A20 knockdown in the WT group, but decreased after A20 knockdown in the miR-21 $1^{-1-}$ group (Fig. 4e). Thus, consistent with the confocal assay, ASC redistribution and oligomerization were suppressed in miR- $21^{-1-}$ macrophages.
miR-21 deficiency inhibits pyroptosis by preventing caspase-1-mediated cleavage of GSDMD

We further examined the role of miR-21 in canonical inflammasome-triggered pyroptosis. In miR-21 $1^{-1-}$ cells, BMDM pyroptosis was blocked upon NLRP3 inflammasome activation triggered by LPS and ATP or nigericin (Fig. 5a). Dying cells presented a typical pyroptosis morphology, with cell swelling and membrane rupture. In contrast, pyroptosis was enhanced in A20-deficient WT and miR-21 $1^{-1-}$ macrophages (Fig. 5a). Because GSDMD is only required for pyroptotic cell death and acts downstream of inflammatory caspases, GSDMD was cleaved in 


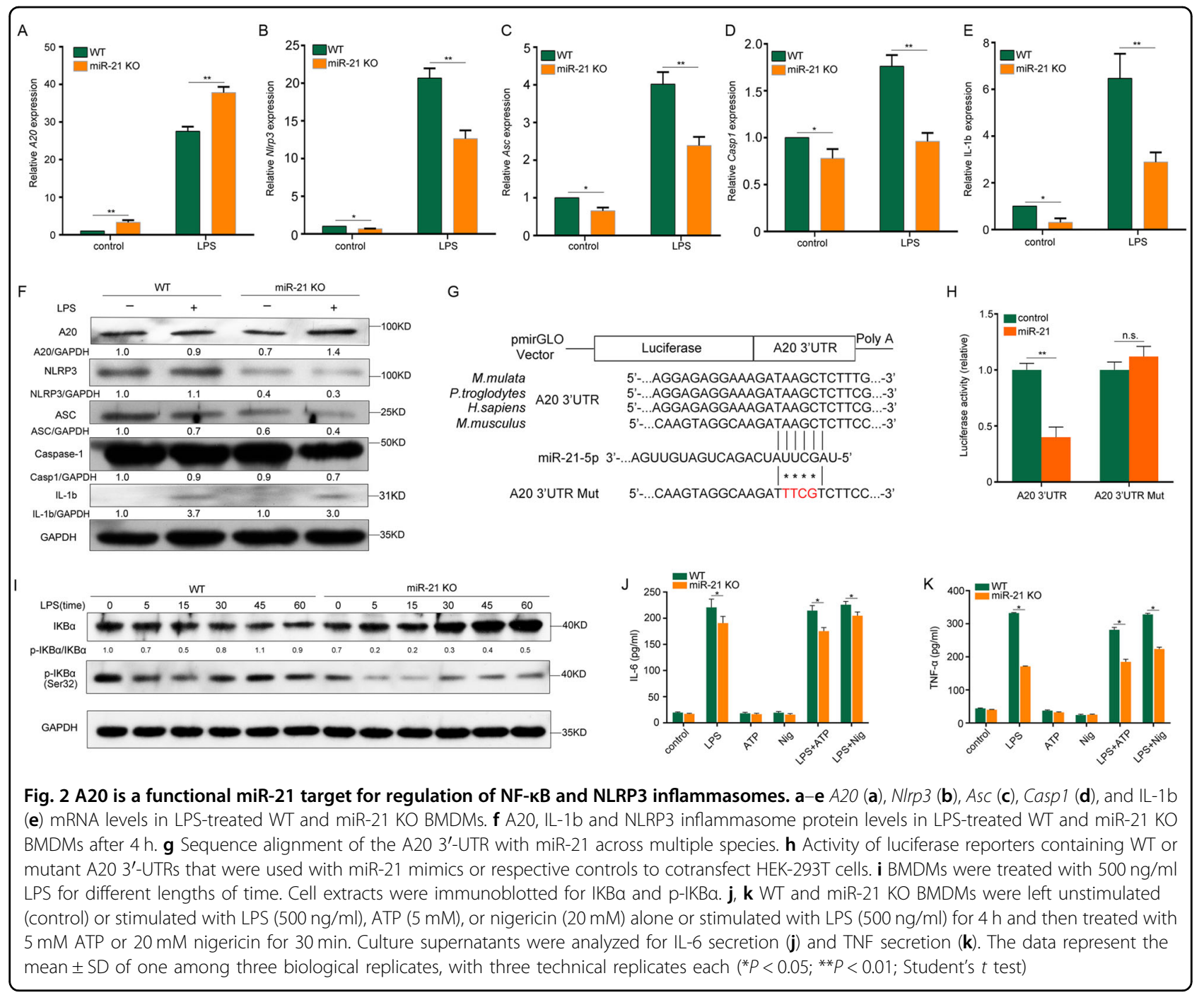

BMDM cells stimulated with LPS plus ATP or nigericin. A cleavage product corresponding to the $\mathrm{N}$-terminal half of Flag-GSDMD was identified, and was decreased compared with the control (Fig. 5b). Similarly, the cleaved GSDMD N-terminus was decreased when miR-21 KO BMDMs were stimulated with LPS plus ATP or nigericin compared with control cells (Fig. 5c). We also found that knockdown of A20 promoted cleavage of GSDMD in WT cells, but this was not observed in miR-21 KO cells (Fig. 5d). These data demonstrate that miR-21 KO inhibits GSDMD cleavage-induced pyroptosis via targeting of A20.

\section{miR-21 deficiency relieves LPS-induced septic shock and organ damage}

To confirm the function of miR-21 in activating NLRP3 inflammasomes in vivo, we studied the role of miR-21 in an LPS-induced septic shock mouse model. The results showed that miR-21 knockout led to much lower mortality rates than those observed in wild-type mice after peritoneal injection of LPS (Fig. 6a). Furthermore, IL-1 $\beta$ and IL-18 secretion levels were significantly decreased in the peritoneal lavage fluid from miR-21-1- mice (Fig. $6 \mathrm{~b}$, c). The proportion of M1 and M2 macrophages was also obviously decreased in peritoneal lavage cells from miR$21^{-1-}$ mice (Fig. 6d, e), and the $\mathrm{M} 1 / \mathrm{M} 2$ ratio was obviously decreased in miR-21 $1^{-1-}$ mice (Fig. 6f). To determine the cause of death, we performed histological analyses of the internal organs. We observed fewer lesions in spleens, kidneys, and livers from miR-21 $1^{-/-}$mice, which also showed decreased inflammatory cell infiltration compared with WT mice (Fig. 6g, h). Collectively, these results demonstrate that miR-21 acts as a critical positive regulator of the $\mathrm{NF}-\mathrm{kB}$ pathway to regulate NLRP3 inflammasome activation and pyroptosis through negative regulation of A20 (Fig. 6i). 


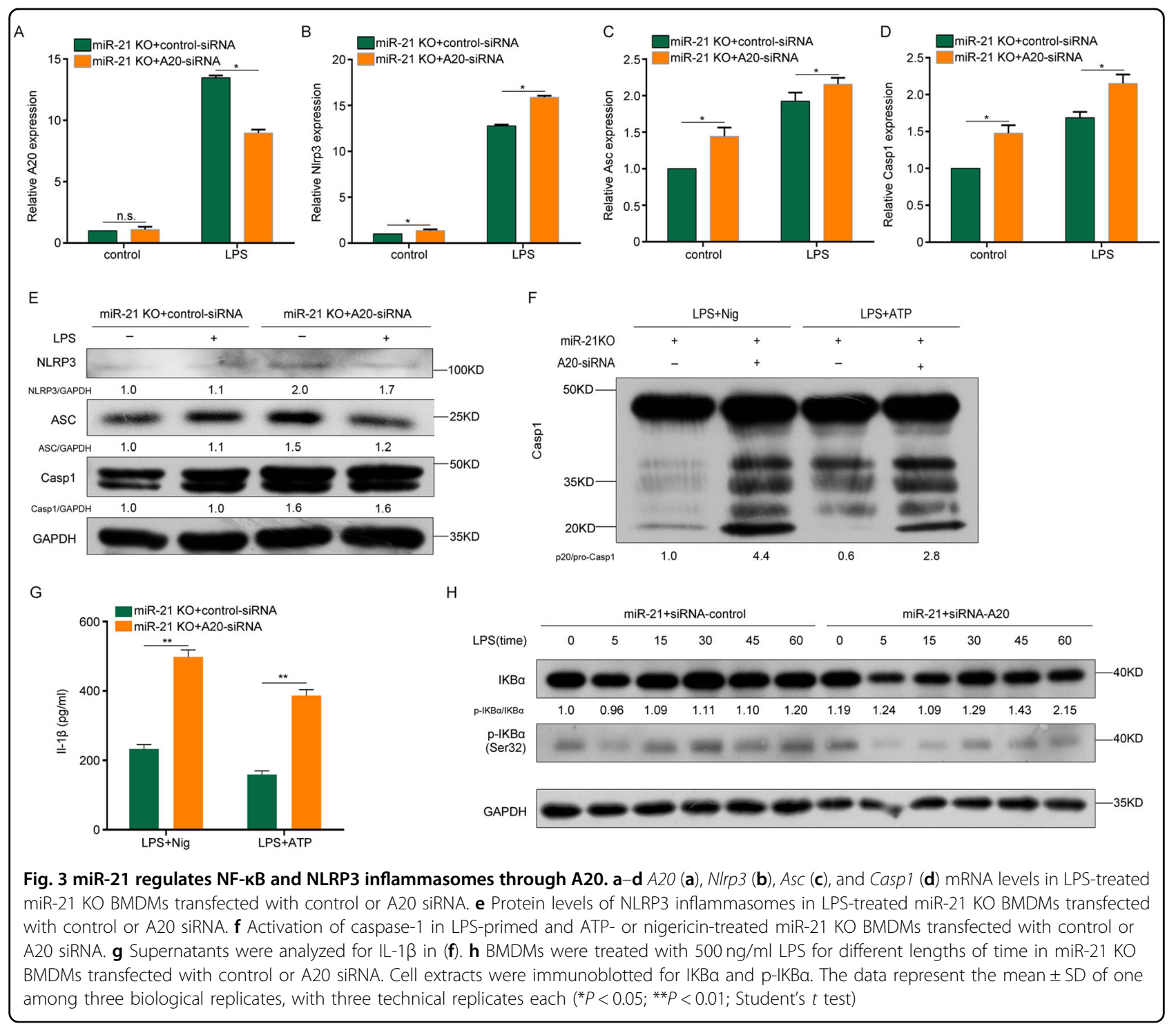

\section{Role of miR-21 in human septic shock}

To evaluate the role of miR-21 in human septic shock, we searched original microRNA expression profile data in the Gene Expression Omnibus (www.ncbi.nlm.nih.gov/ geo/) database for human septic shock. We downloaded GSE26440 expression data of samples from 88 children with septic shock and 26 normal controls from the Gene Expression Omnibus (GEO) database. Differentially expressed miRNAs in samples from patients with septic shock were analyzed in comparison with those in samples from normal controls. We found that miR-21 was obviously upregulated in septic shock patients in the clinical data set (Fig. 7a), including infants $(0.2-1.9$ year, $n=30)$, toddlers $(2.0-5.9$ years, $n=34)$, and school-age children (6-19 years, $n=24)$ (Fig. $7 \mathrm{~b})$. In miR-21 inhibitor-treated PMA-differentiated THP-1 cells, A20 protein levels were upregulated, but NLRP3, ASC, and pro- caspase-1 protein levels were downregulated (Fig. 7c). We next treated LPS-primed PMA-differentiated THP-1 cells with ATP and found that the miR-21 inhibitor blocked caspase-1 activation and cleavage of GSDMD (Fig. 7d, e).

Next, we stimulated human peripheral blood mononuclear cells (PBMCs) from healthy donors with LPS. Consistent with our findings in THP-1 cells, A20 protein levels were upregulated, but NLRP3, ASC, and procaspase-1 protein levels were downregulated (Fig. 7f). We further treated LPS-primed PBMCs with ATP and found that the miR-21 inhibitor prevented caspase-1 activation and cleavage of GSDMD (Fig. $7 \mathrm{~g}, \mathrm{~h}$ ). Therefore, miR-21 is also crucial for regulation of NLRP3 inflammasome activation and GSDMD cleavage in human cells. In general, these data demonstrate that the antiinflammatory effect of miR-21 $\mathrm{KO}$ is conserved between mice and humans. 


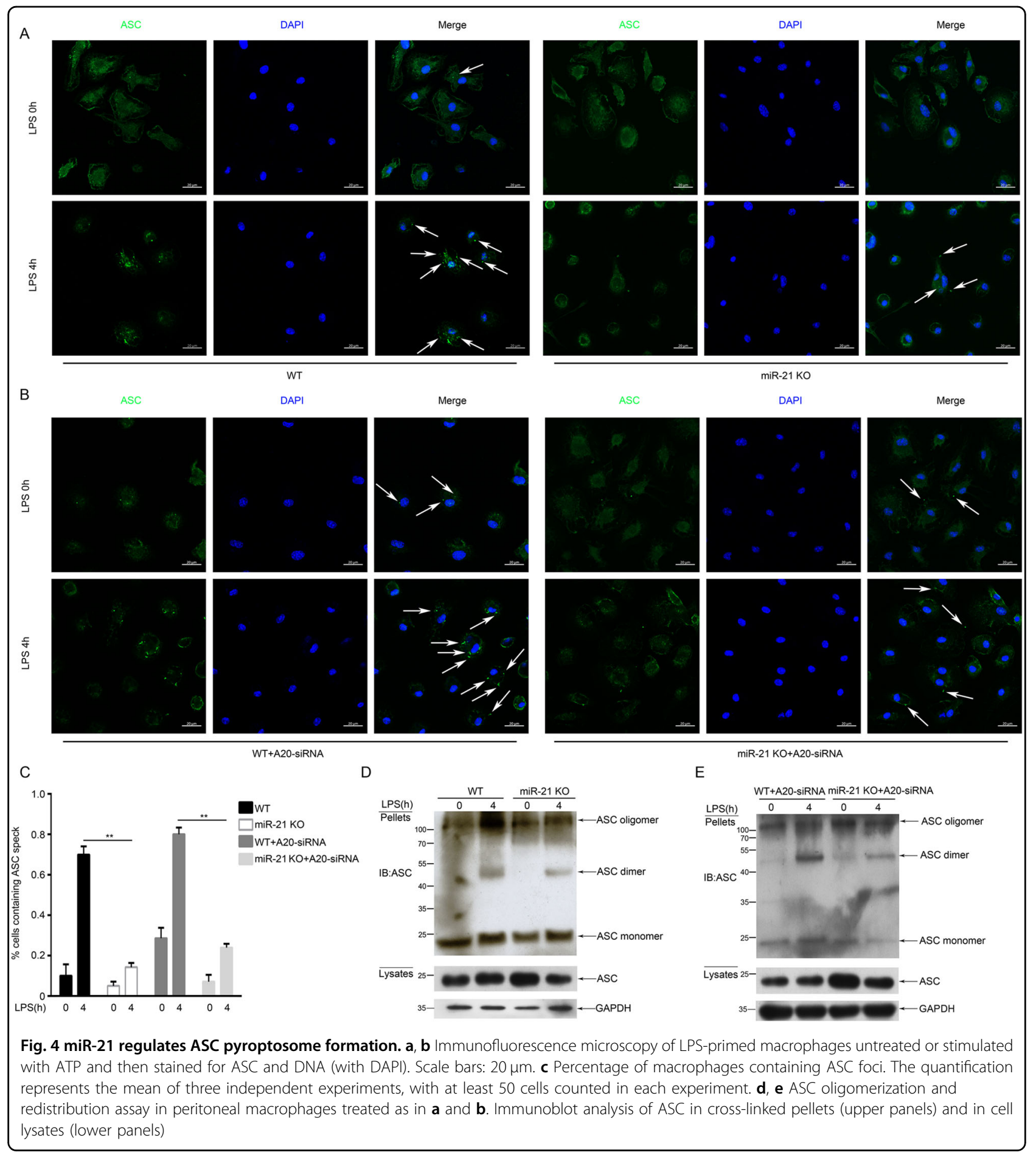

\section{Discussion}

Previous studies have shown that certain miRNAs are abnormally expressed in sepsis patients, and some are associated with decreased survival, supporting their potential use as targeted biomarkers for early sepsis diagnosis and prognosis determination ${ }^{30}$. In addition to its role in various tumor types, miR-21 is aberrantly expressed and plays a role in LPS-induced septic shock ${ }^{21}$, but the mechanisms are still unclear. Our study showed that the proinflammatory effect of miR-21 may result from its ability to promote NLRP3 inflammasome activation, thereby promoting the secretion of IL-1 $\beta$ and cleavage of caspase- 1 and GSDMD. Furthermore, miR-21 mediates IL- 6 or TNF- $\alpha$ production and NLRP3, ASC, 


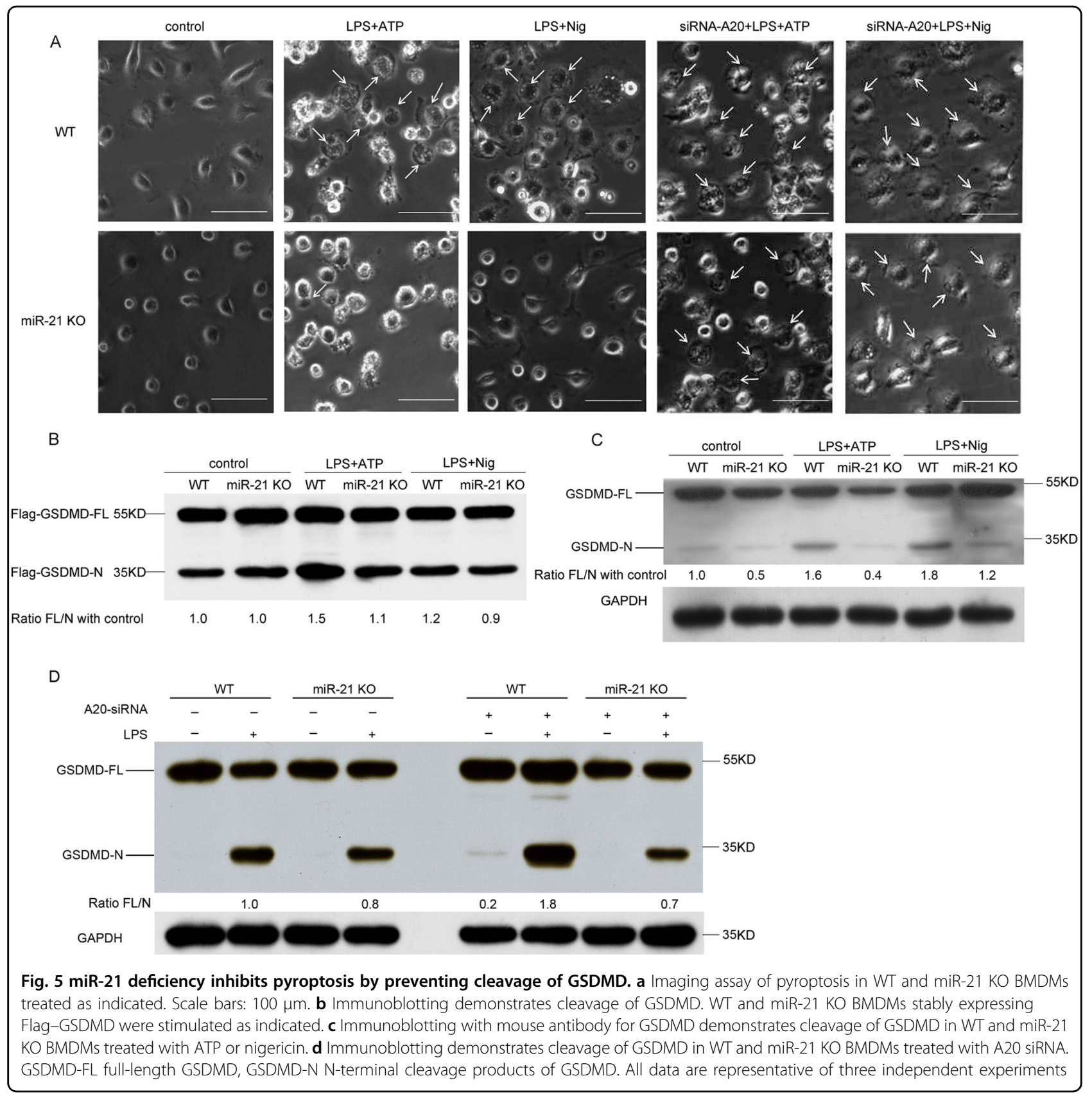

and caspase- 1 expression by targeting A20. Therefore, miR-21 specifically promotes NLRP3 inflammasome activation and the NF- $\mathrm{KB}$ signaling pathway by inhibiting A20. The results also suggest that miR-21 participates in a positive feedback loop to control NF-kB signaling in macrophages.

It has been reported that A20 inhibits NLRP3 inflammasome activation ${ }^{31}$. This study showed that miR-21 deficiency not only prevents IL-1 $\beta$ secretion and caspase1 activation but also prevents caspase-1 cleavage of GSDMD to induce pyroptosis by targeting A20. Moreover, miR-21 deficiency suppresses NLRP3-mediated, but not AIM2-mediated caspase-1 activation. These results suggest that miR-21 knockout can negatively regulate caspase-1 activation through NLRP3 inflammasomes rather than AIM2 inflammasomes. NLRP3 inflammasome activation is strictly controlled to varying degrees in wildtype macrophages. A priming signal (also called the first signal and usually provided by TLR) upregulates Nlrp3 and pro-IL-1b expression levels through the transcription factor NF- $\mathrm{KB}^{22}$, and A20 negatively regulates LPS-induced NF- $k B$ activation ${ }^{4-7}$. We found that miR-21 deficiency negatively regulates LPS-induced NF- $\mathrm{KB}$ activation by promoting A20, leading to reduced secretion of cytokines 


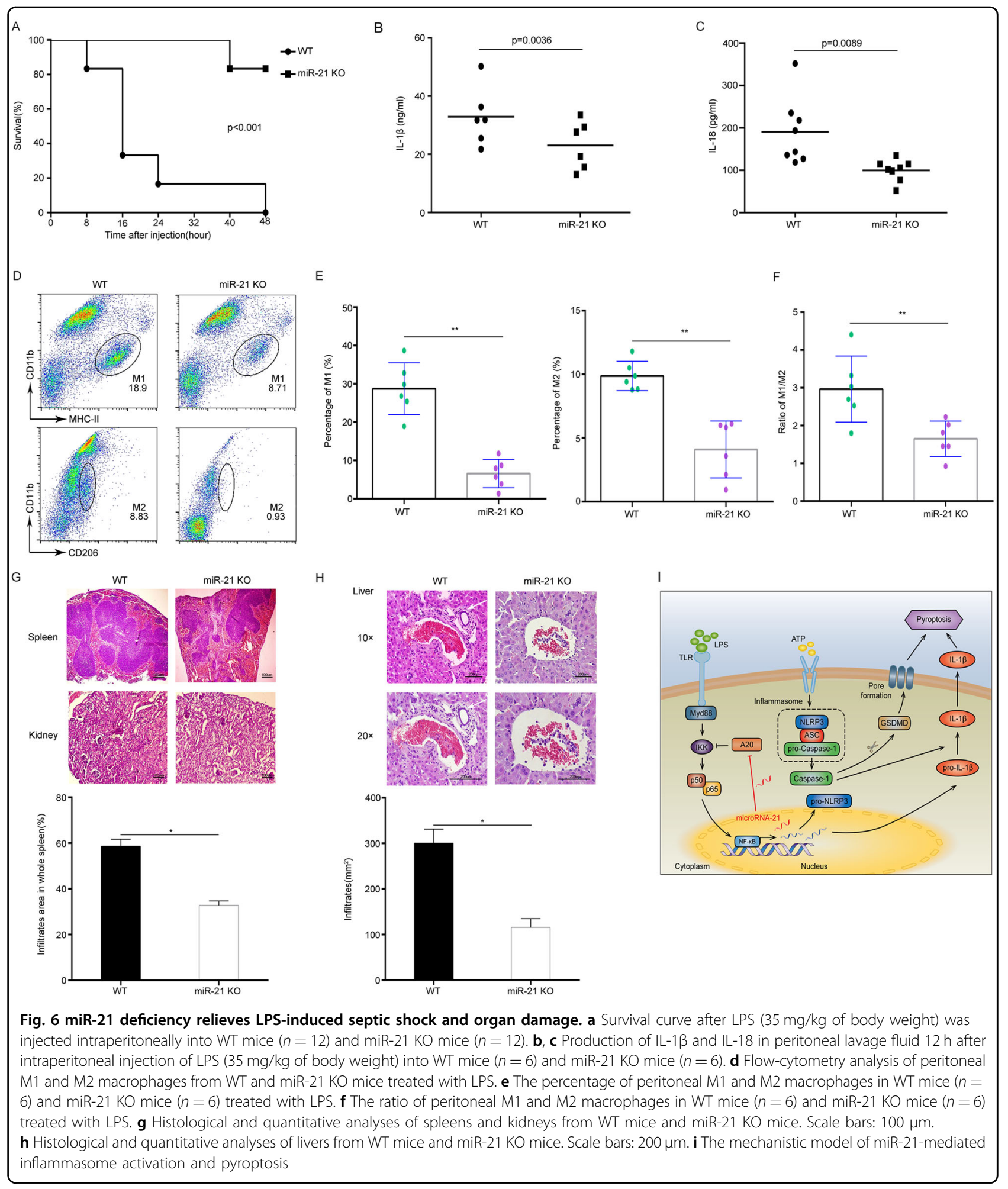

(IL-6 and TNF) dependent on NF-kB in miR-21 KO macrophages. Thus, these data demonstrate that the inhibitory effect of miR-21 deficiency on NLRP3 inflammasome expression is dependent on NF- $\mathrm{KB}$ through promotion of A20. After the priming phase, the second signal is the activation signal, which initiates assembly of several protein complexes, including NLRP3, ASC, and pro-caspase-1, by regulating the formation of the ASC 


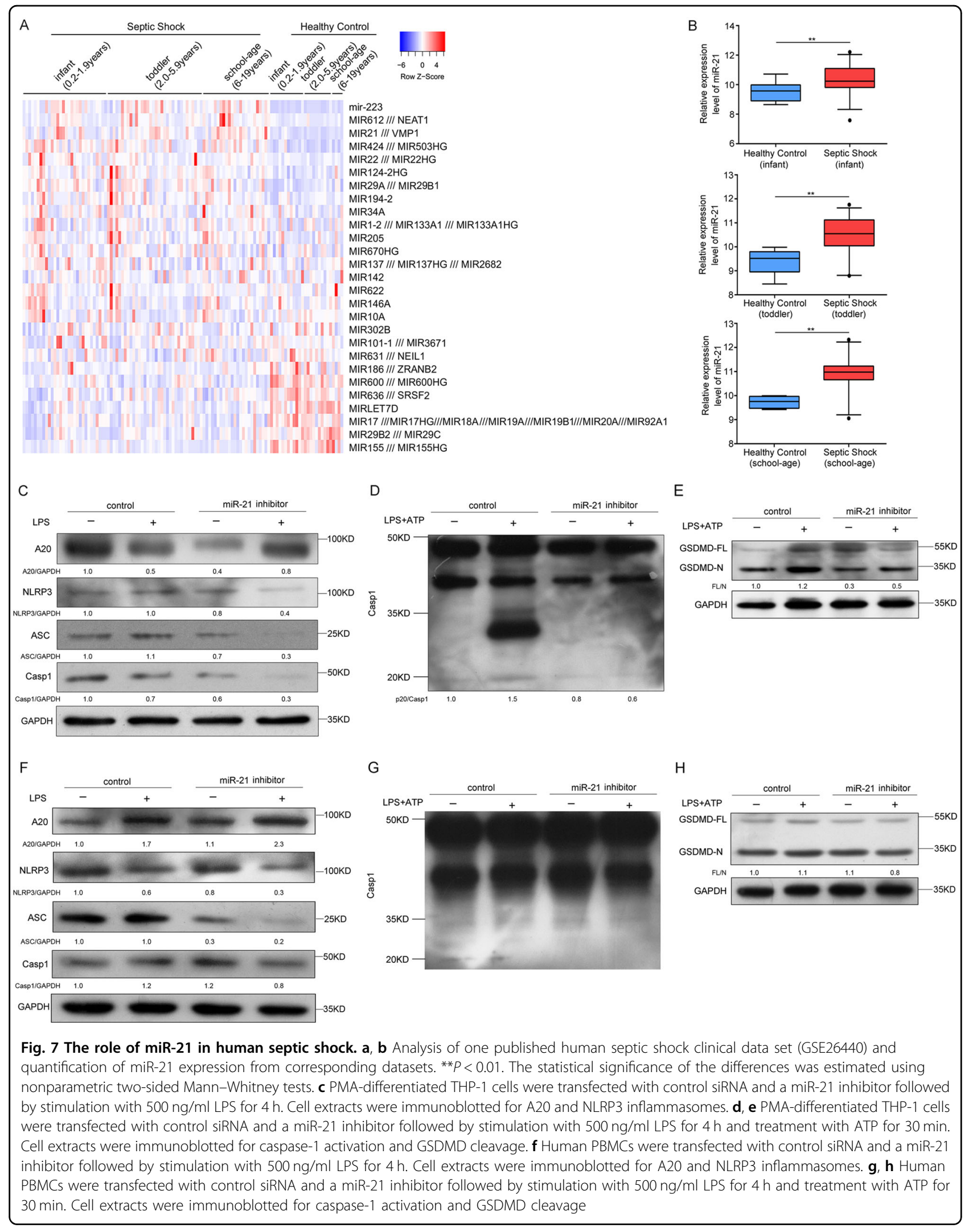


pyroptosome and splicing of caspase- 1 into its active form. In this study, we demonstrated that miR-21 deficiency inhibited ASC oligomerization. Furthermore, we found that A20 knockdown promoted ASC oligomerization in WT cells, but this effect was reversed in miR-21 $\mathrm{KO}$ cells. These data demonstrate that miR-21 regulates ASC pyroptosome formation to activate the NLRP3 inflammasome through targeting of A20.

GSDMD has been identified as a universal substrate for caspase- 1 and caspases-4/5/11. Ligands of various canonical inflammasomes can activate caspase- 1 , while caspases-4/5/11 directly recognizes bacterial LPS, and both activities can trigger pyroptosis ${ }^{32}$. Cleavage of GSDMD by inflammatory caspases critically determines pyroptosis by releasing the cleaved GSDMD-N domain, leading to induction of pyroptosis activity ${ }^{32}$. Despite its crucial role in septic shock, the mechanism of miR-21-induced pyroptosis is unclear. In this study, we found that A20 inhibits the ASC pyroptosome, which induced NLRP3 inflammasome assembly and activation of caspase-1mediated GSDMD cleavage, thus preventing pyroptosis. We further found that miR-21 deficiency reversed the above results through targeting of A20, which prevents NLRP3 inflammasome activation and pyroptosis.

In this study, we found that miR-21 inhibits NLRP3 inflammasome activation in mouse macrophages and human THP-1 cells and primary PBMCs. The decreased activation of caspase- 1 prevents cleavage of GSDMD, thus inhibiting pyroptosis in human THP-1 cells and primary PBMCs with miR-21 knockdown. Hence, miR-21 may act as a novel therapeutic target and biomarker in human septic shock.

In summary, our study provides a mechanistic explanation of miR-21-induced modulation of NLRP3 inflammasome-mediated immune responses and GSDMD-mediated pyroptosis in septic shock. Intriguingly, our results suggest that miR-21 may be an intrinsic positive regulator of abnormal NLRP3 inflammasomeinduced septic shock. We observed that human NLRP3 inflammasomes are inhibited by miR-21 knockdown, suggesting miR-21 as a potential therapeutic target for treatment of inflammatory diseases and septic shock.

\section{Materials and methods \\ Animals}

C57BL/6 mice (6-8 weeks old) were obtained from the Academy of Military Medical Science (Beijing, China). Fei Gao's lab (Institute of Zoology, Chinese Academy of Sciences, Beijing) kindly provided miR- $21^{-1-}$ mice, which were generated using CRISPR-Cas9 technology. These animals were maintained in a specific pathogen-free animal facility at the Experimental Animal Center of Tianjin Medical University (Tianjin, China). The experiments were approved by the Animal Ethics Committee of
Tianjin Medical University (Tianjin, China) and were carried out in accordance with animal care guidelines.

\section{Plasmids and reagents}

Lipopolysaccharide (LPS; E. coli O111:B4, L3024), ATP and nigericin were purchased from Sigma-Aldrich (St. Louis, USA). Cytokines were measured using murine IL-1 $\beta$, IL-18, IL-6, and TNF- $\alpha$ ELISA kits purchased from MultiSciences Biotech (Lianke Bio, Hangzhou, China). Cell death was determined with an LDH assay performed using a CytoTox 96 Non-Radioactive Cytotoxicity Assay kit (Promega, USA). The FUIGW-Flagmgsdmd plasmid was kindly provided by Shao Feng (National Institute of Biological Sciences, Beijing, China).

\section{Cell culture and transfection}

The bone marrow-derived macrophage preparation methods were as follows: bone marrow cells were extracted from femurs and tibias of wild-type and miR$21^{-1-}$ mice. Cells were then cultured at $2 \times 10^{6} \mathrm{cells} / \mathrm{ml}$ in $10-\mathrm{cm}$ cell culture plates in the DMEM supplemented with $10 \mathrm{ng} / \mathrm{ml}$ murine M-CSF. Cells were cultured for 6 days, and fresh medium was added every 3 days. On day 6 , cells were collected for experiments. Macrophages were primed with $500 \mathrm{ng} / \mathrm{ml}$ LPS for $4 \mathrm{~h}$ before stimulation with $5 \mathrm{mM}$ ATP and $20 \mu \mathrm{M}$ nigericin for $30 \mathrm{~min}$. The THP-1 human macrophage line was purchased from the Cell Resource Center of Peking Union Medical College. Cells were cultured in the RPMI-1640 medium supplemented with 10\% FBS (HyClone, GE, USA) and antibiotics $(100 \mathrm{IU} / \mathrm{ml}$ penicillin and $100 \mu \mathrm{g} / \mathrm{ml}$ streptomycin). Human peripheral blood mononuclear cells were prepared as we previously reported ${ }^{33}$. Briefly, $3 \times 10^{6}$ cells were resuspended in $1 \mathrm{ml}$ of RPMI-1640 medium containing $100 \mathrm{IU} / \mathrm{ml}$ penicillin, $100 \mu \mathrm{g} / \mathrm{ml}$ streptomycin, $2 \mathrm{mM}$ L-glutamine, $1 \mathrm{mM}$ pyruvate, and $10 \mathrm{ng} / \mathrm{ml}$ human M-CSF. Cells were cultured for 6 days, and fresh medium was added every 3 days. On day 6 , the cells were collected for experiments. To achieve stable Flag expression, lentiviral plasmids containing the required genes, together with the packaging plasmids pspax 2 and pmd2g, were used to transfect $293 \mathrm{~T}$ cells at a ratio of 5:3:2. After transfection for $48 \mathrm{~h}$ and $72 \mathrm{~h}$, the supernatants were collected and used to infect BMDMs for an additional $48 \mathrm{~h}$. GFP-positive cells were sorted via flow cytometry for subsequent experiments.

\section{Quantitative real-time PCR}

According to the manufacturer's instructions, RNA was extracted using Trizol reagent (Invitrogen, Carlsbad, USA). After RNA purification, contaminated genomic DNA was removed using DNA enzymes. Random hexamers and M-MLV reverse transcriptase (Promega, Madison, USA) were used to reverse transcribe RNAs into 
cDNA. Takara (Takara, Japan) provided all other reverse transcription reagents. Genewiz (Suzhou, China) synthesized the gene-specific primers. In accordance with the manufacturer's instructions, SYBR Green mix (Takara, Japan) was used for relative quantitative real-time PCR. Each reaction was performed on an ABI PRISM 7500 Fast Real-Time PCR System (Applied Biosystems Inc., Foster City, California, USA), and repeated in triplicate. The following primer pairs were used: for mouse Nlrp3, 5'ATTACCCGCCCGAGAAAGG- $3^{\prime}$ and $5^{\prime}$-TCGCAGCA AAGATCCACACAG-3' mouse Asc, 5'-CTTGTCAGG GGATGAACTCAAAA-3' and 5'-GCCATACGACTCCA GATAGTAGC-3' mouse Casp1, 5'-ACAAGGCACGGG ACCTATG-3' and $5^{\prime}$-TCCCAGTCAGTCCTGGAAAT G-3' and mouse Tnfaip3, 5'-GAACAGCGATCAGGC CAGG-3' and 5'-GGACAGTTGGGTGTCTCACATT-3'.

\section{3'-UTR luciferase assays}

The WT or mutant Tnfaip3 3'-UTR was amplified via PCR and cloned into a pmirGLO Dual-luciferase miRNA Target Expression Vector (Promega, USA). Lipo3000 transfection reagent was used to cotransfect 293T cells with miR-21 mimic and pmirGLO Dual-luciferase 3'-UTR vector. At $48 \mathrm{~h}$ post transfection, the cells were harvested and assayed using a dual-luciferase reporter assay system (Promega, USA).

\section{Small interfering RNAs and transfection}

miR-21 mimic, inhibitor, and control siRNA were synthesized by RiboBio (Guangzhou, China). The siRNA for Tnfaip3 knockdown had the following sequence: 5'GCUGUGAAGAUACGAGAGAUU-3' (sense sequence). According to the manufacturer's instructions, cells were treated with siRNAs (final concentration of $25 \mathrm{nM}$ ) and harvested $48 \mathrm{~h}$ after siRNA treatment using Lipofectamine RNAiMAX (Invitrogen, Carlsbad, CA, USA).

\section{Western blot analysis}

Lysis buffer containing $10 \mathrm{mM}$ Tris-buffer (pH 7.6), 1\% Triton X-100, 1\% phosphatase inhibitor cocktail, and $1 \mathrm{mM}$ PMSF was used to lyse cells. Cell lysates were then boiled in SDS sample buffer and resolved on a 10\% SDSPAGE gel. Immunoblots were incubated overnight with primary antibodies against NLRP3 (AG-20B-0014-C100, Adipogen, $\mathrm{CH}$ ), ASC (AG-25B-0006, Adipogen, $\mathrm{CH}$ ), mouse caspase-1 (AG-20B-0042-C100, Adipogen, $\mathrm{CH}$ ), human caspase-1 (AG-20B-0048-C100, Adipogen, CH), IkBa (4814, Cell Signaling, USA), phospho-IkBa (2859, Ser32) (Cell Signaling, USA), A20 (23456-1-AP, Proteintech, USA), IL-1 $\beta$ (12242, Cell Signaling, USA) human GSDMD (sc-81868, Santa Cruz Biotechnology, USA), mouse GSDMD (ab209845, Abcam, USA), and GAPDH (10494-1-AP, Proteintech, USA). Immunoblots were examined using an ECL detection reagent (Millipore Corporation, Billerica, MA, USA).

\section{Flow cytometry}

Cells were washed from the peritoneal cavities of mice injected intraperitoneally with LPS and resuspended in PBS containing 1\% FBS for FACS analysis. According to the manufacturer's instructions, anti-CD11b, anti-F4/80, anti-MHC II, and anti-CD206 antibodies were used for fluorescent staining. Isotype antibody controls were used to exclude nonspecific staining. All flow-cytometric antibodies were obtained from Ebioscience. The data were obtained with a FACSCanto II flow cytometer (BD Biosciences, USA) and analyzed using FlowJo software (Tree star, Ashland, OR).

\section{Microscopy imaging of cell death}

To examine cell death morphology, cells were treated in a 12-well plate according to the instructions for static imaging of live cells. An Olympus IX71 microscope was used to capture static bright-field images of pyroptotic cells. For ASC pyroptosome formation, macrophages were seeded overnight on 35-mm glass-bottom culture dishes. The cells were stimulated and stained with an anti-ASC antibody and DAPI, as described above. After stimulation, the cells were fixed with $4 \%$ paraformaldehyde for $20 \mathrm{~min}$ and then permeabilized with Triton X-100. The cells were then incubated with an anti-ASC antibody for $1 \mathrm{~h}$ and donkey anti-rabbit antibody conjugated to Alexa Fluor 488 as the secondary antibody (Proteintech, USA). Finally, the cells were stained with DAPI. An Olympus FluoView FV1000 microscope equipped with a $\times 60$ objective was used for cell imaging. The images were processed using ImageJ. All image data shown are representative of at least three randomly selected fields.

\section{ASC pyroptosome detection}

As previously reported, we detected the formation of ASC pyroptosomes ${ }^{29}$. BMDMs were cultured in six-well plates at a concentration of $2 \times 10^{6}$ cells $/ \mathrm{ml}$ and treated with different stimulants. Cells were centrifuged and resuspended in $0.5 \mathrm{ml}$ of ice-cold buffer containing $20 \mathrm{mM}$ HEPES-KOH (pH 7.5), $150 \mathrm{mM} \mathrm{KCl,} \mathrm{1 \%} \mathrm{NP-40,}$ $0.1 \mathrm{mM}$ PMSF, and protease inhibitors, and were lysed in a microcentrifuge tube via shearing 20 times with a 21gauge needle. The cell lysate was centrifuged at $5000 \times g$ for $10 \mathrm{~min}$ at $4{ }^{\circ} \mathrm{C}$. The supernatant was collected and diluted with 1 volume of buffer and centrifuged for $8 \mathrm{~min}$ at $5000 \times g$ to granulate ASC oligomers. Next, the resuspended pellets were cross-linked with fresh DSS $(4 \mathrm{mM}$, dissolved in DMSO) for $30 \mathrm{~min}$ and centrifuged for $10 \mathrm{~min}$ at $5000 \times g$. The cross-linked pellets were resuspended in $30 \mu \mathrm{l}$ of SDS sample buffer and separated on a 
10\% SDS-PAGE gel. An anti-mouse ASC antibody was used for western blotting.

\section{Histopathology}

Spleens, livers, and kidneys from wild-type and miR$21^{-1-}$ mice were perfused with $4 \%$ paraformaldehyde infused through the heart and then dissected and fixed overnight. The spinal cord was embedded in paraffin and sectioned $(5-10 \mu \mathrm{m})$ for hematoxylin and eosin staining $(H \& E)$. The degree of inflammatory cell infiltration was analyzed via routine histology and quantified using ImageJ software.

\section{In vivo septic shock model}

To induce cytokine secretion in vivo, 8-week-old female mice were intraperitoneally injected with LPS. At $6 \mathrm{~h}$ after the injection, $0.8 \mathrm{ml}$ of PBS containing 1\% FBS was injected into the peritoneal cavity, and then, the fluid was separated. An ELISA was used to measure the IL-1 $\beta$ level in peritoneal lavage fluid and IL-18 level in serum. To induce septic shock, LPS was injected into the peritoneal cavity of mice, and then the mouse health status was monitored at specific intervals.

\section{Statistics}

GraphPad Prism 6.0 software was used for data analysis. The data are presented as the mean \pm SD of triplicate measurements in a representative experiment. Statistical analysis was performed using an unpaired two-tailed $t$ test. $P<0.05$ was considered statistically significant.

\section{Acknowledgements}

This work was supported by the National Natural Science Foundation of China through nos 81601047, 31600730, 81602496, 81301026, 81302568, and 81541032; the Natural Science Foundation of Tianjin through grant nos 16JCYBJC24800 and 16JCYBJC24600; and the Postdoctoral Foundation of China through grant 2016M591395.

\section{Author details \\ 'Laboratory of Immunology and Inflammation, Department of Immunology, Key Laboratory of Immune Microenvironment and Diseases of Educational Ministry of China, Tianjin Key Laboratory of Cellular and Molecular Immunology, Tianjin Medical University, 300070 Tianjin, China. ${ }^{2}$ Institute of Integrative Medicines for Acute Abdominal Diseases, Nankai Hospital, Tianjin, China. ${ }^{3}$ Department of Genetics, School of Basic Medical Sciences, Tianjin Medical University, Tianjin, China. ${ }^{4}$ Metabolic Disease Hospital \& Tianjin Institute of Endocrinology, Tianjin Medical University, 300070 Tianjin, China. ${ }^{5}$ Institute of Zoology, Chinese Academy of Sciences, 100101 Beijing, China. ${ }^{6}$ Guangdong Province Key Laboratory for Biotechnology Drug Candidates, School of Life Sciences and Biopharmaceutics, Guangdong Pharmaceutical University, Guangzhou, China}

\section{Author's contributions}

Z.X. and R.Z. designed the research. Z.X., H.L., X.G., Z.Z., Q.X., J.Z., G.Y., and D.Z. performed the experiments. Z.Z., Z.X., L.Z., Y.L., H.Y., and Q.Z. performed data analysis. Y.D., C.G., J.Y., S.D., and Z.Y. contributed to experimental guidance. Z.X. and R.Z. wrote the paper. All authors approved the final version of the paper.

\section{Conflict of interest}

The authors declare that they have no conflict of interest.

\section{Publisher's note}

Springer Nature remains neutral with regard to jurisdictional claims in published maps and institutional affiliations.

Received: 18 April 2019 Revised: 23 May 2019 Accepted: 30 May 2019 Published online: 12 June 2019

\section{References}

1. Mariathasan, S. et al. Differential activation of the inflammasome by caspase-1 adaptors ASC and Ipaf. Nature 430, 213-218 (2004).

2. Allen, I. C. et al. The NLRP3 inflammasome mediates in vivo innate immunity to influenza A virus through recognition of viral RNA. Immunity 30, 556-565 (2009).

3. Gross, O. et al. Syk kinase signalling couples to the Nirp3 inflammasome for anti-fungal host defence. Nature 459, 433-436 (2009).

4. Eisenbarth, S. C., Colegio, O. R., O'Connor, W., Sutterwala, F. S. \& Flavell, R. A. Crucial role for the Nalp3 inflammasome in the immunostimulatory properties of aluminium adjuvants. Nature 453, 1122-1126 (2008).

5. Martinon, F., Petrilli, V., Mayor, A., Tardivel, A. \& Tschopp, J. Gout-associated uric acid crystals activate the NALP3 inflammasome. Nature 440, 237-241 (2006).

6. Dostert, $C$. et al. Innate immune activation through Nalp3 inflammasome sensing of asbestos and silica. Science 320, 674-677 (2008).

7. Duewell, P. et al. NLRP3 inflammasomes are required for atherogenesis and activated by cholesterol crystals. Nature 464, 1357-1361 (2010).

8. Man, S. M. \& Kanneganti, T. D. Regulation of inflammasome activation. Immunol. Rev. 265, 6-21 (2015).

9. Lamkanfi, M. \& Dixit, V. M. Inflammasomes and their roles in health and disease. Annu. Rev. Cell Dev. Bi. 28, 137-161 (2012).

10. Bordon, Y. Mucosal immunology: inflammasomes induce sepsis following community breakdown. Nat. Rev. Immunol. 12, 400-401 (2012).

11. Kim, M. J. et al. SESN2/sestrin2 suppresses sepsis by inducing mitophagy and inhibiting NLRP3 activation in macrophages. Autophagy 12, 1272-1291 (2016).

12. Medzhitov, R. Recognition of microorganisms and activation of the immune response. Nature $\mathbf{4 4 9}$, 819-826 (2007).

13. Guo, H. T., Callaway, J. B. \& Ting, J. P. Inflammasomes: mechanism of action, role in disease, and therapeutics. Nat. Med. 21, 677-687 (2015).

14. Wang, L. et al. Human genetic and metabolite variation reveals that methylthioadenosine is a prognostic biomarker and an inflammatory regulator in sepsis. Sci. Adv. 3, e1602096 (2017).

15. Pu, Q. Q. et al. Atg7 deficiency intensifies inflammasome activation and pyroptosis in Pseudomonas sepsis. J. Immunol. 198, 3205-3213 (2017).

16. Hu, Z. S. et al. Antimicrobial cathelicidin peptide LL-37 inhibits the LPS/ATPinduced pyroptosis of macrophages by dual mechanism. Plos ONE 9, e85765 (2014).

17. Tonnus, W. \& Linkermann, A. The in vivo evidence for regulated necrosis. Immunol. Rev. 277, 128-149 (2017).

18. Kayagaki, N. et al. Caspase-11 cleaves gasdermin D for non-canonical inflammasome signalling. Nature 526, 666-671 (2015).

19. Aglietti, R. A. et al. GsdmD p30 elicited by caspase-11 during pyroptosis forms pores in membranes. P. Natl Acad. Sci. USA 113, 7858-7863 (2016).

20. Singer, $M$. et al. The third international consensus definitions for sepsis and septic shock (sepsis-3). Jama-J. Am. Med. Assoc. 315, 801-810 (2016).

21. Wang, $\mathrm{H}$. et al. miR-21-3p controls sepsis-associated cardiac dysfunction via regulating SORBS2. J. Mol. Cell Cardiol. 94, 43-53 (2016).

22. Bauernfeind, F. G. et al. Cutting edge: NF-kappa B activating pattern recognition and cytokine receptors license NLRP3 inflammasome activation by regulating NLRP3 expression. J. Immunol. 183, 787-791 (2009).

23. Wertz, I. E. et al. De-ubiquitination and ubiquitin ligase domains of A20 downregulate NF-kappa B signalling. Nature 430, 694-699 (2004).

24. Vereecke, L., Beyaert, R. \& van Loo, G. The ubiquitin-editing enzyme A20 (TNFAIP3) is a central regulator of immunopathology. Trends Immunol. 30, 383-391 (2009).

25. Lee, E. G. et al. Failure to regulate TNF-induced NF-kappa B and cell death responses in A20-deficient mice. Science 289, 2350-2354 (2000).

26. Boone, D. L. et al. The ubiquitin-modifying enzyme A20 is required for termination of Toll-like receptor responses. Nat. Immunol. 5, 1052-1060 (2004).

27. Oeckinghaus, A. \& Ghosh, S. The NF-kappaB family of transcription factors and its regulation. Cold Spring Harb. Perspect. Biol. 1, a000034 (2009). 
28. Fernandes-Alnemri, T. et al. The pyroptosome: a supramolecular assembly of ASC dimers mediating inflammatory cell death via caspase-1 activation. Cell Death Differ. 14, 1590-1604 (2007).

29. Juliana, C. et al. Anti-inflammatory compounds parthenolide and Bay 11-7082 are direct inhibitors of the inflammasome. J. Biol. Chem. 285, 9792-9802 (2010).

30. Giza, D. E. et al. Cellular and viral microRNAs in sepsis: mechanisms of action and clinical applications. Cell Death Differ. 23, 1906-1918 (2016).
31. Vande Walle, L. et al. Negative regulation of the NLRP3 inflammasome by A20 protects against arthritis. Nature 512, 69-73 (2014).

32. Shi, J. J. et al. Cleavage of GSDMD by inflammatory caspases determines pyroptotic cell death. Nature 526, 660-665 (2015).

33. Xue, Z. et al. Embelin suppresses dendritic cell functions and limits autoimmune encephalomyelitis through the TGF- $\beta / \beta$-catenin and STAT3 signaling pathways. Mol. Neurobiol. 49, 1087-1101 (2014). 